\title{
Responsabilidad Social, Marketing e Industria Farmacéutica: A propósito del informe Mental Health and Integration.
}

Social Responsibility, Marketing and Pharmaceutical Industry: In relation with the report on Mental Health and Integration.

Miguel A. Valverde Eizaguirre a , José A. Inchauspe Aróstegui ${ }^{\text {b }}$

${ }^{a}$ Psicólogo. Unidad de Hospitalización Psiquiátrica Sección A. Complejo Hospitalario de Navarra. Hospital de Navarra. Servicio Navarro de Salud - Osasunbidea. España. ${ }^{b}$ Psiquiatra. Director del Centro de Salud Mental de San Juan. Servicio Navarro de Salud - Osasunbidea. España.

Correspondencia: José A.Inchauspe (jinchaua@cfnavarra.es)

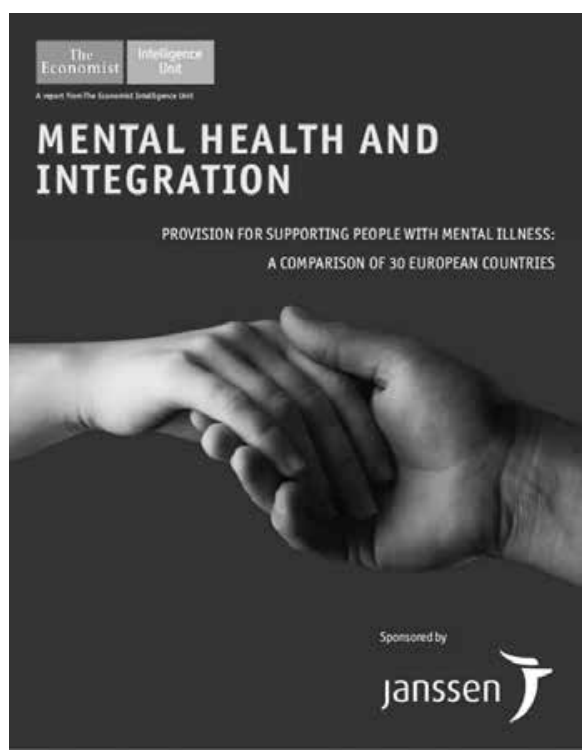

\section{INTRODUCCION}

Mental Health and Integration (1) es un informe realizado por The Economist Intelligence Unit $(\mathrm{EIU})^{1}$ encargado y financiado por Janssen Pharmaceutica. ${ }^{2}$

Este informe se presenta como Libro Blan$\operatorname{co}^{3} \mathrm{y}$ ha sido considerado como ejemplo de responsabilidad social corporativa, un trabajo irrealizable sin la financiación de la industria, y una muestra de su relación colaborativa con las asociaciones científicas. Todo esto justifica un análisis del documento con cierta profundidad.

El objeto del informe es evaluar a "30 países europeos (los 28 de la Unión Europea, más Noruega y Suiza) en la integración en la comunidad de las personas con enfermedad mental" (pg. 2). Presenta un estudio comparativo entre estos países para "contribuir al debate acerca de la integración mostrando las fortalezas y debilidades de cada país concreto, y a partir de ahí el tipo de mejoras necesarias" (pg. 36).

Empresa perteneciente al grupo The Economist. Realiza análisis, informes y previsiones económicas por países, áreas de negocio, industrias y ciudades. En 2012 adquirió Bazian, consultora especializada en temas de salud. Se presenta como una empresa que ayuda sus clientes «a decidir la mejor relación riesgo / beneficio para su negocio.» Cf. http://www.eiu.com/home.aspx

Solo en la relación de colaboradores se informa que el autor es Paul Kliestra. En la introducción indica que EIU es el único responsable de su contenido, que puede no reflejar el punto de vista del patrocinador, aunque también incluye un disclaimer según el cual ni el sponsor ni EIU se responsabilizan de la información, opiniones o conclusiones del texto.

White Paper o Libro Blanco es un informe con autoridad, que pretende ayudar a comprender un tema, afrontar un problema o tomar una decisión (por ejemplo diseñando una política a largo plazo). Los libros blancos se emplean especialmente en dos ámbitos: el gubernamental y el comercial. http://es.wikipedia.org/wiki/Libro_blanco 


\section{EL INFORME}

\section{a. El instrumento de evaluación}

Se crea un instrumento de evaluación -Mental Health Integration Index (Índice de Integración de Salud Mental)- con 18 indicadores encuadrados en cuatro categorías: a) el Entorno (5 indicadores), presencia o ausencia de políticas gubernamentales que fomenten una vida plena para las personas con enfermedad mental, desinstitucionalización, alojamiento, apoyo a familias... b) la Accesibilidad (4 indicadores) a la asistencia y a los servicios sociales, c) las Oportunidades (3 indicadores) asociadas a la búsqueda de empleo, su mantenimiento, y lucha contra la discriminación laboral, y d) los sistemas de Gobernanza (5 indicadores) en referencia a los derechos humanos y la lucha contra el estigma. Todos los indicadores son cualitativos, salvo las tasas de profesionales en servicios de Salud Mental.

El instrumento se completa con otros 7 indicadores: la Discriminación (medición cualitativa de la discriminación de las personas con enfermedad mental), la Tasa de Suicidio, el Gasto en Salud como porcentaje del PIB, el gasto en Salud Mental como porcentaje del gasto en Salud, la Población Total, el PIB y el PIB per cápita. Estos últimos indicadores no puntúan pero sirven para describir un estado de situación, y se asocian al resto para intentar explicar los resultados del ranking.

El informe explica la procedencia de los datos utilizados por el Index, obtenidos cuando ha sido posible de fuentes oficiales, y la metodología utilizada para obtener la puntuación y el ranking, con diversas cautelas y mecanismos de supervisión para asegurar su fiabilidad.

La población objeto del estudio, para la que usa el término personas con "enfermedad mental", ${ }^{4}$ es la de los diagnosticados en cualquiera de las categorías del ICD-10.

Indica que EIU "llevó a cabo una amplia investigación documental e hizo un programa de entrevistas en profundidad con expertos en el tema" (pg.2). El informe no justifica la bibliografía elegida, y cita en el margen del texto principal un número escaso de publicaciones, usadas para respaldar las afirmaciones contenidas en el texto. Tampoco se facilitan criterios de selección de los expertos, ${ }^{5}$ ni de la entrevista realizada. Estas entrevistas no parecen haber sido procesadas en base a alguna metodología, usándose el contenido parcial de las mismas a modo de glosa del texto principal, matizando, desarrollando, enfatizando, explicando y justificando, según los casos, las afirmaciones contenidas en el texto.

"Mental Illness" en la mayor parte del texto.

Son dos paneles diferentes. El primero con 16 expertos. Figuran responsables de agencias gubernamentales de salud, sociales, trabajo, profesores de universidad, clínicos y responsables de centros, especialistas en salud pública, miembros de asociaciones profesionales, mutuas y aseguradoras, de asociaciones de familiares y afectados, investigadores e incluso miembros de "think tanks". Son médicos, psicólogos, terapeutas ocupacionales, otro tipo de técnicos, políticos y familiares de pacientes. El segundo está constituido por expertos que analizan en profundidad la situación de cada país. Colaboran en la evaluación del Index y en alguna ocasión se citan sus opiniones en el texto principal. 
PARA EL DEBATE

\section{b. La estructura del informe}

El informe está constituido por un Resumen Ejecutivo y un capítulo de Conclusiones, dos apéndices, ${ }^{6}$ un disclaimer final, y el cuerpo principal del texto, distribuido en 4 capítulos. No hay apéndice bibliográfico ni declaración de conflicto de intereses. Los 4 capítulos del texto son: 1 "Introducción, la carga de la enfermedad mental en Europa", 2 "Lecciones de los resultados del Index", 3 "Del hospital a la recuperación: Un lento viaje" y 4 "El abismo de los datos".

Los capítulos tienen una estructura similar: se señalan determinados problemas y/o carencias, se ilustran con la opinión de expertos, y se citan publicaciones y trabajos que respaldan los argumentos vertidos.

A cada carencia y/o problema detectado se contrapone, en recuadro, una buena práctica. Para el estigma asociado a la enfermedad mental, el programa británico Time to Change (2), para la integración de servicios y cuidados, los modelos asertivos comunitarios, esencialmente el modelo holandés $F A C T$ (3), para la integración laboral los modelos IPS (4) y la iniciativa alemana $p s y G a,{ }^{7}$ para el apoyo a las familias y cuidadores el proyecto PROSPECT de EUFAMI (5), para incorporar el punto de vista del usuario el PROMs (6) y su aplicación en el Mental Health Recovery Star (7), y para conseguir datos fiables del estado de situación de los cuidados en Salud Mental en Europa el proyecto europeo ROAMER (8).

En cambio el capítulo 2, "Lecciones de los resultados del Index", no selecciona ninguna buena práctica de ningún país respecto a la organización general de servicios de Salud Mental, donde todos presentan carencias, y el recuadro correspondiente lo ocupa la relación entre la porción del PIB dedicada al gasto en Salud Mental y el resultado general del Index, relación que considera más significativo que cualquier otra variable.

No se justifica la selección de estas buenas prácticas entre otras posibles, limitándose a facilitar alguna bibliografía respecto a la eficacia/eficiencia/coste-beneficio de los modelos seleccionados.

c. El contenido del informe

Desde el primer capítulo "Introducción, la carga de la enfermedad mental en Europa" se defiende la existencia de una enorme carga y una respuesta inadecuada, siendo este el argumento sobre el que pivota todo el informe.

\section{La carga}

Indica que el 38\% de europeos (165 millones) se ven afectados cada año por enfermedad mental, con la depresión a la cabeza. Argumenta que los datos del impacto

\footnotetext{
El I con el Ranking del Index. El II describe las peculiaridades del Index y su aplicación.

Una campaña de sensibilización para la integración laboral de personas con enfermedad, en la que han participado mutuas, sindicatos y agencias gubernamentales. Producen documentos, instrumentos de evaluación, organizan seminarios... [Consultado 13-1-2015] Disponible en: http://safety-work.org/en/pages/topics/stress/project_ mental_health_in_the_world_of_work_taking_the_stress_out_of_stress.html
} 
de la enfermedad mental y la discapacidad son en realidad mucho mayores, debido a los suicidios -que se contabilizan al margen, aun siendo indicadores de Salud Mentaly a la gran comorbilidad entre personas con enfermedad mental y otras condiciones médicas por un lado, y otras enfermedades somáticas que cursan con depresión (p.ej. diabetes, problemas cardíacos, pulmonares obstructivos...) por otro.

La esperanza de vida entre quienes padecen una enfermedad mental grave es entre 15 y 25 años menor, aunque rara vez es atribuible a la enfermedad mental propiamente dicha, surgiendo de la combinación de cuidados médicos insuficientes y la tendencia de estas personas a practicar conductas de riesgo (p.ej. fumar).

Informa que en Europa fue la causa del 12\% de años de vida perdidos en 2012 (usando el criterio DALY). ${ }^{8}$ El impacto sobre el PIB se valora en el 3,4\%. Estiman que los datos reales son superiores, teniendo en cuenta los costes indirectos, como pérdida de productividad por incapacidad temporal, etc. Y dado que el $75 \%$ de los casos debutan antes de los 25 años el impacto a largo plazo es superior a otras enfermedades.

La fuente principal de estos datos son dos trabajos $(9,10)$, que comparten buena parte de los autores, publicados en la revista del European College of Neuropsychopharmacology (ECNP). En ambos su perspectiva es la de Disorders of the Brain -Trastornos del Cerebro-, reminiscencia quizás de la Década del Cerebro.

En el primero, Wittchen et al. (2011) (9), preparado para el ECNP y el European Brain Council (EBC), el espectro de trastornos considerados cubre todas las categorías diagnósticas del ICD-10, capítulo V, Salud Mental, incluyendo consumo de sustancias, demencias y retraso mental, y alguna más (hipersomnia, narcolepsia y apnea del sueño). Las más frecuentes son la ansiedad (14\%) y el insomnio (7\%). A lo largo del texto se hacen referencias a otros "trastornos del cerebro" como epilepsia, cefaleas, Parkinson y esclerosis múltiples, ACV, TCE... que si se consideraran junto a los otros la carga de discapacidad aumentaría sensiblemente. No se incluyen estos datos por problemas de tipo metodológico y solapamiento (comorbilidad) entre unos y otros. Se insiste en que ambos (trastornos mentales y neurológicos) son "trastornos del cerebro". Consideran los autores que su aproximación a la prevalencia es conservadora y evita la doble contabilidad por comorbilidad. Afirman que los criterios diagnósticos muestran auténticos "trastornos" con relevancia clínica y no simples "problemas de salud mental", al incluir (según los manuales DSM-III y IV) criterios de duración, gravedad y repercusión funcional. Critican el escepticismo ante estas cifras de prevalencia, atribuyéndolo a conocimiento limitado, actitudes negativas, y errores conceptuales sobre la naturaleza del trastorno mental. Estiman la carga de discapacidad en un 26,6\% del total, incluyendo aquí ciertos problemas neurológicos (ACV, epilepsia, parkinson, migrañas y esclerosis múltiple, por ejemplo).

8 En español AVAD (años de vida ajustados por discapacidad). Los AVAD suman los años de vida perdidos (AVP) debido a la mortalidad prematura y los años perdidos por enfermedad/discapacidad (APD). 
PARA EL DEBATE

El segundo, Gustavsson A, et al (2011) (10), encargado por el European Brain Council (EBC), comparte el marco conceptual de "trastornos del cerebro" e incluye en sus cálculos el daño cerebral por TCE, epilepsia, ACV, esclerosis múltiple, parkinson, cefaleas, y tumores cerebrales. Defiende tal inclusión dado que los trastornos mentales y los neurológicos comparten substrato cerebral, y porque ambas disciplinas, a pesar de obedecer a tradiciones diferentes, comparten métodos y abordajes. Estima que solo el $37 \%$ de los costes es atribuible al coste sanitario directo, afirmando ser conservadores en sus estimaciones, pudiendo subestimar el coste de algunas patologías y en algunos países.

Ambos trabajos detectan mayor prevalencia en sus revisiones de 2010 comparada con las del 2005, atribuibles a considerar más categorías diagnósticas y una mayor franja de edad -en el estudio de Wittchen (9), a niños y ancianos.

\section{Una débil respuesta}

El informe afirma que la respuesta al problema es insuficiente e inadecuada, dado que solo un cuarto de los afectados es tratado, y adecuadamente aún menos, lo que representa un "reto ético terrible" (pg.9).

Los datos proceden también del artículo de Wittchen (9), que refiere el retraso en el inicio del tratamiento, que la inmensa mayoría son tratados en atención primaria, y que solo el $10 \%$ de personas reciben un tratamiento correcto, sea con fármacos o psicoterapia, según los mínimos contemplados en las guías clínicas. Concluye que hay necesidades importantes sin cubrir, y que la carga asociada a los "trastornos del cerebro" ha sido subestimada en Europa. El aumento de la esperanza de vida significa que el problema tiende a crecer. La prevención y el tratamiento de los "trastornos del cerebro" serán el reto nuclear del siglo XXI, siendo necesario realizar investigación básica en psicoterapia y terapia farmacológica, en salud pública y en etiopatogenia. Consideran inadecuado centrarse únicamente en los trastornos más severos y comórbidos, dado que los problemas comienzan a una edad temprana - en la infancia o en la adolescencia-, raramente remiten por si solos, y se complican a lo largo de la vida en gravedad, comorbilidad y repercusión funcional. Se debe cambiar por tanto la diana de las intervenciones a edades tempranas. Los costes de estos trastornos no son tanto directos (tratamiento) como indirectos (bajas, incapacidades laborales...). Se propone aumentar los costes directos para disminuir los indirectos. El artículo de Gustavsson et al., ratifica esas conclusiones y solicita a la industria farmacéutica que detenga "su tendencia a abandonar la investigación" respecto a los psicofármacos.

La existencia de esta brecha del tratamiento es reforzada apoyándose en otros artículos citados en este capítulo referidos al estigma (11), en el capítulo 2 al explicar que en Alemania, que lidera el ranking global del Index, casi la mitad de personas con 
enfermedad mental grave no recibe tratamiento médico específico ${ }^{9}(12)$, desemboca en el capítulo 3 presentando el Tratamiento Asertivo Comunitario (ACT) ${ }^{10}$ como modelo asistencial a generalizar, abogando por el modelo holandés FACT.

En el capítulo, "Débil respuesta", se pone en labios de expertos que los tratamientos disponibles no son siempre satisfactorios. "Ninguno recupera totalmente (...) aunque aplicar los actuales puede marcar diferencias Muchas personas pueden funcionar independientemente hasta cierto grado y algunas pueden recuperarse completamente por largos periodos" (Insel, pg. 10). Para Wittchen el progreso ha tenido lugar en los últimos veinte años... y afirma que si la aplicación de estos tratamientos y estrategias se extiende tendrá efectos en la incidencia y prevalencia de la enfermedad (pg. 10).

d. Las conclusiones del informe

El informe concluye en las siguientes recomendaciones:

o Disponer de mejores datos. Los datos epidemiológicos, de los procesos asistenciales médicos y sociales y de medida de resultados son escasos o inexistentes.

o Suministrar una financiación adecuada a Salud Mental.

o Finalizar la tarea de décadas de desinstitucionalización.

o Proporcionar servicios integrados, basados en la comunidad. Se concreta en la implantación y generalización de equipos ACT.

o Incluir la prestación de servicios integrados dirigidos al empleo.

Tres de las recomendaciones hacen referencia a ampliar la población a tratar y una a mejorar los resultados mediante la integración de servicios.

e. Lo que el informe no considera

\section{i. Los puntos ciegos del informe}

Además de la pobreza de referencias bibliográficas ${ }^{11} \mathrm{y}$ otros aspectos metodológicamente dudosos, como la selección de buenas prácticas, y la opacidad en el tratamiento de las entrevistas y los párrafos seleccionados en el informe ${ }^{12}$, se pueden considerar algunos "puntos ciegos", aquellos aspectos soslayados de los artículos que sustentan el informe, o los reflejados en la opinión de los entrevistados, que no se res-

$9 \quad$ En Mak S. et al (2014) (12) Wittchen figura como último firmante y Jacobi como segundo, ambos coautores de los trabajos citados antes, Wittchen et al. (2011) (9) y Gustavsson et al. (2011) (10). El artículo remite constantemente a otros trabajos de Wittchen. Concluye que aunque se desarrollen servicios completos y no existan barreras financieras, el uso de los servicios ni es mayor ni ha cambiado, por ejemplo en el lapso de tiempo entre la aparición de los primeros síntomas hasta la búsqueda de ayuda.

10 Diseñado para captar aquellas personas con trastorno mental grave más reticentes a frecuentar servicios de Salud Mental.

11 Por ejemplo, para la brecha terapéutica, base principal del argumentario, el informe se apoya en el mismo círculo de autores, Wittchen, Jacobi, Jacobson... No se consideran otras referencias y tampoco la literatura crítica con el actual mainstream en Salud Mental

12 Se entrevista al menos a un representante de organizaciones muy críticas con el estado actual de los tratamientos en salud mental, MIND, p.ej., sin mostrar sus opiniones en esta área. Tampoco se recogen las opiniones de Thomas Insel críticas respecto a los manuales diagnósticos al uso. 
PARA EL DEBATE

paldan con datos y/o artículos, no obstante fácilmente accesibles. Se muestran seguidamente algunos:

- Sobre la esperanza de vida entre quienes padecen una enfermedad mental, uno de los textos citados dedica un capítulo entero a la morbimortalidad debida el impacto de los medicamentos, en particular de los antipsicóticos, y entre estos los de última generación, del litio y del valproíco, así como de la polifarmacia. Refiere los resultados obtenidos en el estudio CATIE "mucho menos influenciado por la industria farmacéutica que estudios previos" (13).

- Respecto al estigma, que el informe considera como la "principal barrera" de acceso a los servicios de Salud Mental (pg. 11), el artículo citado como respaldo (14) lo considera la cuarta "barrera en la búsqueda de ayuda profesional" y su influencia sería de pequeña a moderada. Las dos primeras barreras serían "de lejos" el deseo de solucionar el problema por uno mismo, y la falta de conciencia de necesitar ayuda. Otro de los artículos citados (11) recoge opiniones parecidas, y añade la creencia de que los tratamientos psiquiátricos no sean efectivos, el desacuerdo e insatisfacción con el tipo de tratamiento facilitado, en general, y realiza una mención especial a las personas que tras ser sometidas a Terapia Electroconvulsiva evitan el contacto con servicios de Salud Mental. Muestra también la preferencia de muchos usuarios por servicios de psicoterapia (facilitada por médicos o psicólogos) respecto a otros.

- Respecto a los Tratamientos Asertivos Comunitarios (ACT), el artículo citado debate su posible dimensión coercitiva y paternalista, y sobre el tratamiento involuntario. Considera inconsistente su aplicación a todas las personas diagnosticadas de trastorno mental grave. Subrayan la adherencia a la medicación y su administración "in vivo" como características cruciales del modelo, cuyos resultados serían mejores en la disminución de la sintomatología que en la funcionalidad. Finalmente critican un modelo de programas dirigidos únicamente a monitorizar la toma de medicación, "como ya ha sucedido en USA" (15).

El informe tampoco trata otras cuestiones referidas por algunos entrevistados, a pesar de su relevancia:

- La falta de efectividad de los tratamientos (Thomas Insel, pg. 10).

- El abordaje negativo de la enfermedad mental por profesionales más preocupados por la estabilidad clínica que por la recuperación (Kevin Jones, de EUFAMI, pg.11).

- Se señala "cierto clima de hostilidad entre los interesados, más allá de las habituales disputas médicas sobre la definición y tratamiento de ciertas enfermedades". Lo indica el testimonio de una experta, que pasó de representar a pacientes con Enfermedad de Parkinson -relaciones positivas paciente-clínico- al mundo de Salud Mental, que es "totalmente diferente, en el que algunos líderes de pacientes 
tenían en mala consideración a los médicos debido a sus tratamientos pasados, ya que algunos padecieron electroshock y otros habían sido contenidos. La relación médico-paciente no es una en la que se debate y se llega a acuerdos. Es una batalla." (Mary Baker del European Brain Council, pg. 12).

\section{Lo que se soslaya del debate actual en Salud Mental.}

El informe soslaya, como si no existieran, cuestiones candentes en la situación actual, como las que siguen, que resultan obligadas al realizar un estudio de situación y proponer recomendaciones asistenciales.

- La discapacidad asociada a los problemas de Salud Mental. El que las tasas de discapacidad sean mayores en los países con mejor acceso al tratamiento, con mayor uso de psicofármacos, bajo las prácticas clínicas dominantes, y con las políticas laborales habituales de nuestro entorno, es una posibilidad que se está cargando de pruebas.

- El caso de Dinamarca, situado en lo más alto del ranking de países según el Index, puede ser un ejemplo. Es el tercero en la puntuación global y también es el país más regular en todas las áreas. El informe considera a Dinamarca como ejemplo de lo que se consigue manteniendo el esfuerzo adecuado en el área de salud mental. Pero en la figura 1 vemos que el número de casos nuevos de discapacidad por año se ha duplicado en una década. El que esto ocurra en un sistema de calidad no se ha considerado, pero requiere indagación y reflexión. Resulta posible que también ocurra en otros países (16). En USA en los veinte años previos a 2007 creció la tasa de discapacidad, desde 543 personas discapacitadas a 1315 por 100.000 habitantes -entre los menores se multiplicó por 35.(17) Seguramente saber de la discapacidad y sus causas en Salud Mental resulta prioritario, más que clonar un sistema en más sitios.

- La práctica clínica habitual y su respaldo científico. El científico danés Peter Gøtzsche ha abordado públicamente este tema. Ofrece datos, al igual que otros autores, (17-21) que limitan el uso farmacológico, resalta la posible nocividad de la práctica dominante actual, con polifarmacia, y su uso a largo plazo. Gøtzsche muestra un análisis centrado en diez temas, asuntos claves en la asistencia psiquiátrica habitual, incluyendo además la mala ciencia y la corrupción científica en este campo (22). 
Figura 1.

Nuevos casos de discapacidad debido a enfermedad mental en Dinamarca

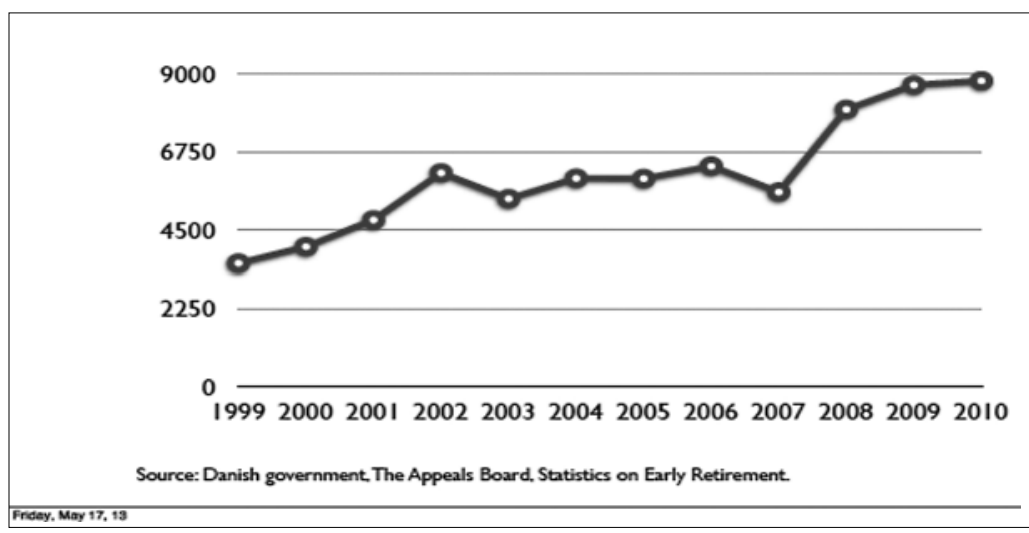

- Sobre el estigma, la marginación y la valoración de los usuarios. El informe hace una referencia relativamente extensa a la campaña antiestigma, Time to change (Tiempo de cambiar), en el Reino Unido, según los informes realizados por MIND, sin referir su especificidad que incluye cierto cuestionamiento: "olvida las etiquetas, simplemente escúchame" dice uno de los lemas. MIND, una de las organizaciones que implementan la campaña, ha realizado numerosos estudios sobre la valoración que hacen los pacientes del sistema asistencial y sus prácticas en UK, que aquí no se tienen en cuenta, aunque sean algunos de los mejores estudios sobre el estigma percibido y la asistencia desde la perspectiva del paciente.

El informe soslaya las preferencias de los pacientes sobre el tratamiento, a pesar de numerosos estudios al respecto. Existe un movimiento de usuarios considerable, con unos 600 grupos en el Reino Unido. que habla de la brecha entre lo que necesitan y lo que se les ofrece o se les obliga a tomar (23). El informe recoge la opinión de asociaciones de familiares subvencionadas por la industria, pero no las de los propios pacientes, como Hearing Voices Network.

- El sobrediagnóstico, la sobremedicación, la medicalización creciente, y la flexibilización de los criterios diagnósticos que permiten incluir a más personas como candidatas a tratamiento, tampoco ha sido tenido en cuenta. Así, por ejemplo, en USA, y no será muy diferente en Europa, un estudio sobre la población afirma que más del $24 \%$ de las personas padecen un trastorno bipolar (24). Sin considerar la inflación de diagnósticos en el S. XXI no se puede realizar un informe con pretensión de diagnóstico de situación y realizar recomendaciones sobre política asistencial.

- Sobre el tratamiento involuntario. Tampoco se muestra en absoluto la situación del tratamiento coercitivo y el internamiento involuntario aunque afirma que es uno de los criterios del Index. 
PARA EL DEBATE

\section{LA NATURALEZA DEL INFORME}

El informe no cumple con el rigor exigible a un White Paper. Tiene más bien la forma de reportaje, fácil de leer, ilustrado con opiniones diversas, y no exento de interés por la información facilitada sobre las llamadas buenas prácticas, y sobre los distintos países, y sus regiones.

Lo que resulta más patente del documento es la estructura retórica de la argumentación:

Primero defiende que existe una condición insuficientemente o inadecuadamente tratada.

Lo segundo es la dimensión catastrófica del problema. La enorme pérdida de potencial vital y laboral en numerosas áreas de sufrimiento: estigma, los suicidios, la carga familiar, y para las naciones, etc. Se subraya la dimensión del fracaso.

La tercera área argumental se centra en lo que se podría conseguir con un cambio. El objetivo actual no es la reducción de síntomas, sino la recuperación, una vida plena, con integración laboral y social. Es el espacio para las historias de éxito.

La cuarta área es la necesidad de darse como objetivo identificar y tratar a más personas, tras indicar que existen formas mejores de asistir que en el pasado. Se trata de movilizar las prácticas, mostrando líneas estratégicas para superar barreras para que los futuros pacientes sean tratados. Se insiste, entre otras medidas, en implementar y generalizar programas de $\mathrm{ACT}$, una intervención inicialmente diseñada para tratar pacientes poco interesados en el tratamiento.

Son cuatro de los cinco principios argumentales del marketing farmacéutico en salud mental. El quinto consiste en defender que la condición a tratar es de tipo biológico, que solo será correctamente tratada con los avances farmacológicos. En este informe es prescindible, pero el promotor, y las demás empresas del ramo, ya interviene habitualmente en esta línea, tanto sobre los profesionales como sobre los usuarios y sus familias. Aún con todo el informe defiende suavemente esta línea argumental indicando que las condiciones a tratar son "enfermedades mentales" equivalentes a enfermedades del cerebro.

Otro aspecto clave del marketing farmacéutico consiste en crear un mensaje coral. Se trata de mostrar a muchos participantes en la elaboración y difusión de su contenido, en este caso son unos treinta colaboradores, pertenecientes a una docena de países, con personalidades de prestigio, y diversas asociaciones de científicos, familiares y otros organismos (su enumeración ocupa dos páginas). El documento está salpicado con voces que subrayan el relato del informe. Esta forma coral del mensaje ayuda a reducir la resistencia a la persuasión del receptor.

El informe indica la participación de figuras relevantes de numerosas instituciones públicas y privadas. El grueso de las organizaciones cuenta con importante subvención de la industria farmacéutica como European Brain Council (EBC), European Federation of Associations of Families of People with Mental Illness (EUFAMI), Global 
PARA EL DEBATE

Alliance of Mental Illness Advocacy Networks-Europe (GAMIAN - Europe), y European Mental Health Systems Network (EHMA) entre otras. ${ }^{13}$ GAMIAN, EUFAMI y EHMA también forman parte del panel asesor del informe.

El promotor de este tipo de actividad se encarga de presentar la información como si fuera elaborada por otros y se resguarda en un discreto segundo plano. Actuando de esta forma, es fácil que los intereses del promotor no sean inmediatamente percibidos, y no aparece tan vinculado al contenido de la información facilitada y las líneas de actuación propuestas (25). Cuenta a su favor la dificultad de los profesionales para detectar y comprender esta modalidad de marketing, sus mecanismos de influencia y distinguirlo de la información.

\section{COMENTARIOS FINALES}

Este informe es un documento que pertenece al campo del marketing farmacéutico, tanto por lo que muestra, como por lo que soslaya y por lo que impulsa. Los datos mostrados, su selección y su ordenación están siempre a favor del promotor, y oculta aquello que siendo igualmente importante o fundamental podría no beneficiarle. El interés de la industria farmacéutica incluye abrir nuevos campos comerciales, con más pacientes a tratar. Por ello, las situaciones asistenciales que puedan detectar a más pacientes pueden resultarle atractivas e idear estrategias de diversos tipos para impulsarlas.

Lo que resulta claro es que un mayor compromiso de los Gobiernos Europeos en Salud Mental en la perspectiva del informe, desde un primer momento, incrementará los beneficios de la industria que proporciona el tratamiento.

Como explica en su lema de presentación, The Economist Intelligence Unit, ayuda a las organizaciones de salud a construir y mantener un negocio exitoso y sostenible, definiéndose como una empresa de marketing ${ }^{14}$ (29).

Seguramente las empresas farmacéuticas serían más útiles para los clínicos realizando estudios rigurosos de uso a medio y largo plazo sobre eficacia y seguridad de sus productos, algo que el campo de Salud Mental necesita para poder recomendar responsablemente los tratamientos a largo plazo.

13 Cf. las paginas web de las asociaciones.

European Brain Council (EBC) http://www.europeanbraincouncil.org/pdfs/Accounts/EBC\%20Accounts\%202004. pdf; http://www.europeanbraincouncil.org/membership/

European Federation of Associations of Families of People with Mental Illness (EUFAMI) http://www.eufami.org/ test/6-eufami-annual-report-2013

European Mental Health Systems Network (EHMA) http://www.ehma.org/index.php?q=node/1091

Global Alliance of Mental Illness Advocacy Networks-Europe (GAMIAN - Europe) http://gamian.eu/wp-content/ uploads/2014/06/Gamian-Europe-Annual-report-2013.pdf

14 Cf. http://www.eiu.com/home.aspx 


\section{BIBLIOGRAFÍA}

(1) The Economist Intelligence Unit "Mental Health and Integration. Provision for supporting people with mental illness: a comparison of 30 European countries" 40 pgs, 2014 [Consultado 1-4-2015] Accesible en http://www.janssen.ie/sites/stage-janssen-ie.emea.cl.datapipe. net/files/FINAL\%20WHITE\%20PAPER\%20-\%20PHIRPSY10140001_v1.0.pdf

(2) Time to Change [Consultado 13-1-2015] Disponible en: http://www.time-to-change. org.uk/

(3) van Veldhuizen JR, Bähler M. "Manual Flexible ACT Vision, model, practice and organization" Groningen, 2013.

(4) Individual Placement and Support. [Consultado 13-1-2015] Disponible en: http:// waamh.org.au/development-and-training/individual-placement--support-ips.aspx

(5) EUFAMI. Prospect. [Consultado 13-1-2015] Disponible en: http://www.eufami.org/ resources-services/prospect

(6) Patient Reported Outcome Measures. [Consultado 13-1-2015] Disponible en: http:// www.hscic.gov.uk/proms

(7) Outcomes Star. Mental Health Recovery Star ${ }^{\mathrm{TM}}$ [Consultado 13-1-2015] Disponible en: http://www.outcomesstar.org.uk/mental-health/

(8) ROAMER A roadmap for mental health research in Europe. [Consultado 13-1-2015] Disponible en: http://www.roamer-mh.org/

(9) Wittchen HU, Jacobi F, Rehm J, Gustavsson A, Svensson M, Jönsson B, et al. The size and burden of mental disorders and other disorders of the brain in Europe 2010. European Neuropsychopharmacology (2011) 21, 655-679.

(10) Gustavsson A, Svensson M, Jacobi F, Allgulander C, Alonso J, Beghi E, et al. Cost of disorders of the brain in Europe 2010. European Neuropsychopharmacology (2011) 21, 718-779.

(11) Thornicroft G, Rose D, Mehta N. Discrimination against people with mental illness: what can psychiatrists do? Advances in psychiatric treatment (2010), vol. 16, 53-59.

(12) Mack S, Jacobi F, Gerschler A, Strehle J, Höfler M, Busch MA, et al.Self-reported utilization of mental health services in the adult German population -evidence for unmet needs? Results of theDEGS1-MentalHealthModule (DEGS1-MH). Int. J. Methods Psychiatr. (2014) Res. 23(3): 289-303.

(13) Mauer B. Morbidity and Mortality in People with Serious Mental Illness. National Association of State Mental Health Program Directors (NASMHPD) Medical Directors Council $13^{\text {th }}$ Technical Reports, 2006.

(14) Clement S, Schauman O, Graham T, Maggioni F, Evans-Lacko S, Bezborodovs N, et al. What is the impact of mental health-related stigma on help-seeking? A systematic review of quantitative and qualitative studies. Psychol Med. 2015 Jan;45(1):11-27.

(15) Rosen A, Mueser KT, Teesson M. Assertive community treatment-Issues from scientific and clinical literature with implications for practice. J Rehabil Res Dev. 2007;44(6):813-26.

(16) Whitaker R. Rethinking Psychiatric Care History, Science, and the Long-term Effects of Psychiatric Drugs. Presentación 2013. [Consultado 13-1-2015] Disponible en: https://www. madinamerica.com/wp-content/uploads/2011/11/Anatomy-of-a-Global-Epidemic-antipsychotics.pdf

(17) Whitaker R. Anatomy of an Epidemic: Magic Bullets, Psychiatric Drugs, and the Astonishing Rise of Mental Illness in America EEUU: Broadway Books, 2010. 
PARA EL DEBATE

(18) Bentall R.P. Medicalizar la mente ¿Sirven de algo los tratamientos psiquiátricos? Barcelona: Herder, 2011.

(19) Moncrieff, J. The Myth of the Chemical Cure: A Critique of Psychiatric Drug Treatment. UK: Palgrave Macmillan, 2009.

(20) Jackson, G.E. Drug-Induced Dementia: a perfect crime. EEUU: AuthorHouse, 2009.

(1) Davies, J. Cracked: The Unhappy Truth about Psychiatry. UK: Pegasus Publications, 2014.

(21) Gøtzsche, P. La psiquiatría se hunde (traducción) (2014). [Consultado 13-1-2015] Disponible en: http://www.nogracias.eu/2014/08/10/la-psiquiatria-se-hunde/

(22) Coles S, Keenan S, Diamond B. Madness Contested: Power and Practice. UK: PCCS Books, 2013

(23) Maturo A. Bipolar disorder and the medicalization of mood: an epidemics of diagnosis? en Mukherjea, A. (editor) Understanding emerging epidemics: Social and political approaches. UK: Emerald, 2010 p 225-242.

(24) Valverde MA. El arte de hacer visible lo invisible: el marketing en el TDAH. (2015). Aceptado para publicación en NORTE de Salud Mental. 